lumbar puncture was performed before the $\mathrm{CT}$, and CT was performed on the day of the headache, lumbar puncture may have been performed too early to detect blood degradation products in the CSF and therefore too early to distinguish a traumatic tap from a genuine haemorrhage. In the absence of degradation products the patient may have had a non-haemorrhagic cause of the headache.

With regard to figure 2, a slice of the CT made after the second episode of headache, it would be interesting to know the interval between the onset of symptoms and the CT. Perimesencephalic haemorrhage can be diagnosed reliably only if the initial CT is performed within 3 days after onset of the symptoms. After this interval distinction between aneurysmal and perimesencephalic patterns becomes unreliable.

The second issue is that of long term outcome. On long term follow up the authors found a high rate of persisting symptoms such as headaches, irritability, depression, and fatigability. This contrasts with the good quality of life (as measured by the sickness impact profile, a validated questionnaire on quality of life) found in a follow up study performed in The Netherlands. ${ }^{4}$ If the methods used by Marquardt et al are valid, the difference in outcome between these two study populations requires an explanation. This is where the question on management strategy for patients with perimesencephalic haemorrhage comes in. Do the authors include any restriction in counselling their patients who have had a perimesencephalic haemorrhage, or are such restrictions imposed on these former patients by physicians who assess people before employment or by medical advisors of insurance companies? We reassure patients on discharge and again a couple of weeks later, at an outpatients, consultation, that a perimesencephalic haemorrhage is not a warning for a major neurological event, we do not impose any restrictions, and we stimulate patients to take up all activities they undertook before the haemorrhage. We hypothesise that imposing restrictions or sharing uncertainties or worries with patients can lead to subjective symptoms as described above. Given this possibility we are reluctant to start informing patients that perimesencephalic haemorrhages can reoccur, as long as recurrences have not been reported convincingly. We do agree that the patient reported on forms a diagnostic challenge, but we have too little evidence to agree that this patient has had two episodes of perimesencephalic haemorrhage.

G J E RINKEL

Department of Neurology, University Medical Centre, PO Box 85500, 508 GA Utrecht, The Netherlands

B K VELTHUIS Department of Radiology

Correspondence to: Dr G J E Rinkel

1 Marquardt G, Niebauer T, Schick U, et al. Long term follow up after perimesencephalic subarachnoid haemorrhage. $\mathcal{f}$ Neurol Neurosurg Psychiatry. 2000;69:127-30.

2 Rinkel GJE, Wijdicks EFM, Vermeulen M, et al. Non-aneurysmal perimesencephalic subarachnoid hemorrhage: $\mathrm{CT}$ and MR patterns that differ from aneurysmal rupture. AfNR Am $\mathscr{f}$ Neuroradiol 1991;12:829-34.

3 van der Wee N, Rinkel GJE, Hasan D, et al. Detection of subarachnoid haemorrhage on early CT: is lumbar puncture still needed after a negative scan? $₹$ Neurol Neurosurg Psychiatry 1995;58:357-9.

4 Brilstra EH, Hop JW, Rinkel GJE. Quality of life after perimesencephalic haemorrhage. $\mathcal{F}$ Neurol Neurosurg Psychiatry 1997;63:382-4.
The authors reply:

We respond to some of the questions raised by Rinkel and Velthuis on our recent publication in this fournal. ${ }^{1}$ The patient of interest presented with typical clinical signs of subarachnoid haemorrhage. He complained of sudden onset of severe headaches, irradiation into the nuchal region, and nausea. Lumbar puncture was performed and blood stained CSF was found. Centrifugation of the CSF disclosed xanthochromia of the supernatant fluid and cytology demonstrated siderophages indicating the presence of intracranial haemorrhage as no lumbar puncture was carried out earlier. ${ }^{2}$ Non-contrast enhanced CT showed blood in the ambient cisterns and these findings were interpreted as perimesencephalic subarachnoid haemorrhage in two different hospitals.

Four vessel digital subtraction cerebral angiography with multiple views was negative as was a repeated angiography 10 weeks later. A third angiography performed in the course of the second episode of haemorrhage again did not disclose any source of the bleeding, and thus the causes of bleeding remain unresolved. A recent publication by Canhao et al studied the prevalence of vascular risk factors in patients who had perimesencephalic subarachnoid haemorrhage. ${ }^{3}$ They found that hypertension was more frequent among patients with perimesencephalic haemorrhage than among two control groups and that among women, smoking was more common in perimesencephalic haemorrhage. However, the medical history of our patient was not relevant, and there was no history of previous arterial hypertension.

Rinkel and Velthuis express their concern about a high rate of persisting symptoms such as headaches, irritability, depression, and fatiguability in long term follow up of our patients. They state that these findings contrast with the good quality of life found in a follow up study performed by Brilstra $e t a l^{4}$ and that these differences require explanation.

In this study, which was cited by us as well, quality of life was measured by means of the sickness impact profile and outcome of patients with perimesencephalic subarachnoid haemorrhage was compared with that of a reference population. Analysing the submitted data, however, significant differences towards less dysfunction in patients were proved only for the categories body care, movement, and household management. Six of the 25 patients $(24 \%)$ had more dysfunction in the category work than the reference population, and 11 patients $(44 \%)$ reported a change in their headache pattern as non-specific headaches occurred more often than before the haemorrhage in 10 patients and less often than before in one patient. Two patients reported fear of rebleeding. Brilstra et al concluded that patients with a perimesencephalic haemorrhage have no reduction in quality of life but had to admit "that most consequences of the perimesencephalic subarachnoid haemorrhage are found in the psychosocial domains." They relate the problems with short term memory, sleeping, fears, irritability, and nervousness with the haemorrhage itself and with the experience of sudden illness leading to admission to an intensive care unit. ${ }^{4}$ These results imply that in the Dutch study as well persisting symptoms are frequent and this does not contrast with our findings at all. However, the focus of our follow up study was directly on these psychosocial implications of perimesencephalic subarachnoid haemorrhage. Only $38 \%$ of our patients thought that they were fully recovered and completely well whereas $62 \%$ of the patients had residual complaints. Moreover, only $41 \%$ of the patients returned to their previous occupation whereas $53 \%$ of the patients retired from work and one man became unemployed. Thus quality of life after the haemorrhage is not as excellent as suggested and it becomes obvious that perimesencephalic subarachnoid haemorrhage has an enormous impact on individual patients and social life.

We do agree with Rinkel and Velthuis on the further management strategy for patients with former PMSAH. We inform the patients of the benign nature of the disease and do not impose any restrictions at all. We also reassure the patients that they can return to the same regular daily activities they undertook before the haemorrhage.

It is supposed that in $15 \%$ to $20 \%$ of the patients with subarachnoid haemorrhage the angiogram is negative and that patients with PMSAH account for about half of these patients with angiogram negative subarachnoid haemorrhage. ${ }^{35}$ On these premises there must be thousands of patients every year who are treated for PMSAH throughout the world. However, reviewing the literature in 1996, Schwartz and Solomon could only find 169 reported patients who had PMSAH. ${ }^{5}$ It seems, therefore, reasonable to compile more data to gain more information about the natural course of PMSAH in significantly larger cohorts of patients.

G MARQUARDT T NIEBAUER U SCHICK R LORENZ Neurosurgical Clinic, fohann Wolfgang Gothe-University, Schleusenzveg 2-16, 60528 Frankfurt am Main, Germany

Correspondence to: Dr G Marquardt

1 Marquardt G, Niebauer T, Schick U, et al. Long term follow up after perimesencephalic subarachnoid haemorrhage. 7 Neurol Neurosurg Prachnoid haemorrhage. F

2 Vermeulen M, van Gijn J. The diagnosis of subVermeulen $M$, van Gin J. The diagnosis of sub-
arachnoid haemorrhage. $\mathcal{I}$ Neurol Neurosurg arachnoid haemorrhage. ${ }^{7}$
Psychiatry 1990;53:365-72.

3 Canhao P, Falcao F, Pinho E, et al. Vascular risk factors for perimesencephalic non-aneurysmal subarachnoid hemorrhage. f N Neurol 1999;246: $492-6$

4 Brilstra EH, Hop JW, Rinkel GJE. Quality of life after perimesencephalic haemorrhage. $\mathscr{f}$ Neurol Neurosurg Psychiatry 1997;63:382-4.

5 Schwartz TH, Solomon RA. Perimesencephalic non-aneurysmal subarachnoid hemorrhage: review of the literature. Neurosurgery 1996;39: 433-40.

\section{Idiopathic intracranial hypertension and anticardolipin antibodies}

The study by Kesler $e t$ al ${ }^{1}$ concludes with the assumption that the presence of anticardiolipin antibodies (aCL-Abs) indicates a unique subgroup of patients with idiopathic intracranial hypertension. Their study does not support this view. They regard as important in this respect the fact that the three patients with aCL-Abs $(p<0.035)$ were significantly older than those without. This is hardly surprising when it is known that the incidence of these antibodies increases with age and may be identifiable in up to $12 \%$ of healthy people. ${ }^{23}$ Their control group therefore needs to be age matched. Further speculation for 
this conclusion is in their statement that there may have been an occult thrombosis of the cerebral venous sinuses, a fact that I agree with as CT and MRI cannot exclude a thrombosis for certain-hence intracranial hypertension would not be the diagnosis. It is not stated how soon cerebral sinus imaging was performed after the onset of symptoms. Thirdly, the concentration of raised aCL-Ab in these patients is not very high apart from the initial measurement in patient 1 . Titres less than 40 units are generally not thought to be pathological but this is quite an arbitrary figure as test systems are variable and not standardised. ${ }^{4}$ Fourthly, with a prevalence of aCL-Ab at $5 \%$ in Israel, the presence of these antibodies in three of 37 patients is not a significant finding $\left(\chi^{2}\right.$; $\mathrm{p}>0.45)$. Finally, it is an accepted view that the presence of aCL-Ab may represent an epiphenomenon due to a non-specified injury. This is supported by the incidental findings of aCL-Ab in symptom-free patients. ${ }^{5}$ Hence the findings cannot support the authors' proposal that the patients with $\mathrm{aCL}-\mathrm{Ab}$ form a subgroup of patients with intracranial hypertension.

O C BACKHOUSE

Department of Ophthalmology, Leeds General Infirmary, The Leeds Teaching Hospitals NHS Trust, Leeds LS1 3EX, UK

Correspondence to: Dr O Backhouse obackhouse@hotmail.com

1 Kesler A, Ellis M, Reshef T, et al. Idiopathic intracranial hypertension and anticardolipin antibodies. If Neurol Neurosurg Psychiatry 2000;68:379-80.

2 Vila P, Hernandez M, Lopez-Fernandez M, et al. Prevalence, follow up, and clinical signifcance of the anticardolipin antibodies in normal subjects. Thromb Haemost 1994;72: 209-13.

3 Fields R, Toubbeh H, Searles R, et al. The prevalence of anticardolipin antibodies in a prevalence of anticardolipin antibodies in a
healthy elderly population and its association healthy elderly population and its association with antin.

4 Biousse V. Coagulation abnormalities and their neuro-ophthalmic manifestations. Curr Opin Ophthalmol 1999;10:382-93.

5 Greaves M. Antiphospholipid antibodies and thrombosis. Lancet 1999;353:1348-53.

\section{BOOK REVIEWS}

The Neuropathology of Schizophrenia. Progress and Interpretation. Edited by PAUl J HARRISON and GARETH $w$ ROBERTS (Pp374, £65.00). Published by Oxford University Press, Oxford, 2000. ISBN 0-19-262907-7.

The neuropathology of schizophrenia has been for a long time perhaps one of the most controversial fields of biomedical research. In the middle decades of the last century there was an increased interest in the neuropathology of psychosis based on the assumption that structural alterations in the brain would provide insight into the understanding of this complex and devastating disease. However, the results of these investigations have been contradictory and it has become a cliché to say that schizophrenia is the graveyard of neuropathologists. Indeed, the results of neuropathological investigations were confusing, and resulted from both clinical and pathological problems. The clinical definition of schizophrenia has been controversial and for a long time internationally accepted diagnostic criteria did not exist. Patients' cohorts were extremely variable and clinical histories far from complete. Most patients had treatment which again varied from centre to centre. The neuropathological methodology was also somewhat primitive and inappropriate to detect subtle changes. Moreover, the material examined varied considerably from centre to centre and sometimes the pathological changes described were the result of another disease process, including epilepsy or minor traumas. It was thus not surprising that these studies were contradictory.

With the advent of neuroimaging a new era has started. It was Johnstone and her colleagues who showed structural alterations (enlarged ventricles) in the brain of psychotic patients using at that time, the novel methodology of CT scanning. Not much later a report of Stevens observing astrocytosis in the brains of patients with schizophrenia, rekindled interest in the neuropathology of schizophrenia.

This book is a comprehensive review of cerebral changes associated with psychosis. The 15 chapters cover a wide range of structural, functional, macroscopical, histological, neurochemical, and pharmacological changes associated with the disease. In addition there are chapters on animal models and methodological issues, as well as on the consequences of treatment. The results of structural and functional imaging are reviewed, the second in relation to neural circuitries. There is an excellent chapter on cerebral asymmetry, a feature important in the understanding of the disease. Two chapters deal with development, one more specifically with cortical development, giving a concise review of the molecular basis for the organisation of the forebrain and pattern formation in relation to pathogenesis. Synaptic pathology and the organisation of cortical circuitries, for a long time inaccessible to conventional methodology, have become the subject of intense research, and recent developments have been suitably summarised in two separate chapters. The chapter on cortical pathology reviews a new generation of quantitative microscopical studies in relation to the GABA, glutamate, and dopamine systems. The problems of gliosis are revisited in a separate chapter with the conclusion that it is unlikely to be a core feature of the neuropathology of schizophrenia. A chapter examines schizophrenia from the perspective of other neurodegenerative diseases and lesions, including those which may cause schizophrenia-like symptoms-for example, metabolic diseases, epilepsy, and psychosis in neurodegenerative disorders. These provide useful information in the differential diagnosis of schizophrenia and other diseases of the nervous system with similar symptomatology.

This is a timely book, reviewing recent developments in our understanding of the disease mechanism in schizophrenia. The editors have brought together international experts in the field to produce a book with a true multidisciplinary approach. Their achievement should be congratulated. However, less praise should be lavished on the publishing house, which has failed to invest in high quality reproductions. There is a single coloured plate of neuroimaging of MRI and PET and the black and white reproductions of the same images have not been removed.
None the less, this is a book which should be purchased by those who are interested in schizophrenia, both neuropathologists and psychiatrists.

P L LANTOS

Philosophical and Ethical Problems in Mental Handicap. By PETER BYRNE (Pp175, $£ 40.00)$. Published by Macmillan Press, Basingstoke, 2000. ISBN 0312234600.

I cannot recommend this slim monograph highly enough to anyone working with people who have the disorders of neurological development that give rise to what we term mental handicap, mental retardation, or learning disability. It is tightly argued, well written, and thought provoking and bears reading much more than once.

Psychiatrists are still the dominant medical profession working with people with learning disability, a fact of history that has been slow to change. Lothian has recently closed its large institution, one of several in Scotland that had around 1000 beds at its peak, with ward sizes to match. It is easy to condemn this partitioning of a whole section of society; it is much more difficult to reconcile and accept the fact that they do differ in a host of ways from most people and yet at the same time they have identical moral worth to any other human being. Professor Byrne's central tenet is that these are not mutually exclusive concepts, but can be-indeed must beintegrated to make philosophical sense. The other paths lead on the one hand to the concept that the labelling process has created a fictional disability, that its use is a method of social control, of maintaining power by the creation of an underclass, and this denial of intrinsic or primary disability is inherent in some of the concepts of full inclusion and normalisation. The other position, perhaps more worrying, is held by some philosophers of bioethics who would define humanity and moral worth on the ability to reason, placing people with cognitive disability in a separate domain in which it is permissible to use eugenic policies to select against them, to justify infanticide.

Byrne argues with great care against both these paths, his arguments grounded on the commonality of humanity and what it is to be human, carefully dissecting and dismissing the accusation that this opens the way to the charge of speciesism. He progresses from the definitions of mental handicap, the moral status of the disabled, through the difficult terrain of euthanasia, abortion, genocide, and oppression, finally ending with theological interpretations that offer alternatives to humanism.

An appreciation of a common humanity is the best protection for such people. I have usually found that the persons are not so concerned as to whether we describe their intrinsic cognitive condition as a mental handicap or a learning disability but as to whether we remember their first and second names.

Psychiatrists may think that of course we know all this, and that of course we would not follow any of those extreme paths that are described by Professor Byrne. The 86 men who used to be herded together in one of the old wards that I inherited might not agree.

WALTER MUIR 